\title{
Comparing local and remote motion aftereffects
}

\author{
MICHAEL voN GRÜNAU and STÉPHANE DUBÉ \\ Department of Psychology, Concordia University, 1455 de Maisonneuve Boulevard West, Montréal, \\ Québec H3G IM8, Canada
}

Received 23 October 1991; revised and accepted 7 April 1992

\begin{abstract}
A new method, using phase-reversing sinusoidal gratings to cancel perceived motion, was developed to measure the motion aftereffect (MAE). This technique was used to show the existence of a remote MAE, i.e. an MAE in areas that were not directly stimulated during adaptation. In several experiments, this remote MAE was compared to the local MAE. The remote effect was generally weaker and of shorter duration. It showed no directional tuning within the investigated range, as compared to a tuning of $\pm 60 \mathrm{deg}$ of the local MAE. There was no adaptation effect to the component gratings of a plaid, indicating that the plaid was treated as a coherent pattern. The local MAE showed clear spatial frequency tuning, whereas the remote MAE varied little with spatial frequency difference, although there was a tendency towards frequencies lower than the adaptation frequency. The possibility is considered that both local and remote MAEs are generated in extrastriate areas.
\end{abstract}

\section{INTRODUCTION}

The negative motion aftereffect (MAE) or waterfall illusion refers to the perception of illusory motion in a direction opposite to that of a previously viewed adapting stimulus. Like many other aftereffects, the MAE can be measured easily only in those retinal areas that have also been directly exposed to the adapting stimulus (Anstis and Gregory, 1965). The delineation between adapted (with MAE) and non-adapted (no MAE) regions is rather sharp. These facts are consistent with a conceptualization that requires that adaptation and test stimuli excite the same neurons, and that these neurons have relatively small receptive fields (RFs) with well-defined borders. Optimal stimuli for these units would have to be placed in a specific location within the RF and would typically cover most of the RF (simple-cell-like arrangement). This kind of framework would suggest the primary visual area V1 as a place with appropriate neural characteristics. Thus this area has often been assumed to be the primary site for the MAE, though evidence on the absolute or relative locus of the MAE is still controversial (Lehmkuhle and Fox, 1975; Anstis and Duncan, 1983; Wiesenfelder and Blake, 1991). Austis and Duncan have suggested that MAEs occur at different levels as a result of adaptation of monocularly or binocularly driven pathways.

There have been some indications, moreover, that MAEs can also be observed outside the adaptation area. In one approach, Weisstein et al. (1977) observed a negative MAE to a moving phantom grating, produced by the motion of surrounding gratings. In another approach, von Grünau (1986) measured a negative MAE in the space between the two stimulus flashes of a stroboscopic motion setup. In these instances, however, a motion stimulus was perceived in the test area during adaptation, even if no direct physical stimulation had occurred. Attempts to measure an MAE in an area that, during adaptation, has received neither physical nor 'perceptual' stimulation have been rather unsuccessful. Wohlgemuth (1911) studied some 
such displays, but his test stimuli were not restricted to the non-stimulated area, and, therefore, induced motion with MAEs cannot be excluded as an explanation.

Thus, the existence of MAEs in non-stimulated areas has not yet been tested adequately. Critical for the detection of such MAEs might be the kind of stimulus used during the test phase. It only needs to be demonstrated that a directional bias exists for one particular direction over its opposite. Tasks and stimuli used most often in the past, like detection of apparent motion of a stationary version of the adaptation stimulus, or cancellation of the MAE by a stimulus moving in the opposite direction, may have been too insensitive for more subtle directional biases in remote test areas (i.e. areas other than the adaptation area) to emerge. In the following series of experiments, therefore, we used a more subtle test stimulus, namely the perceived direction of an ambiguous phase-reversal stimulus (Levinson and Sekuler, 1975) as an indicator for the presence of the MAE. It is more subtle in the sense that motion in both the adaptation direction and the opposite direction are present at all times.

In the experiments reported below, we first demonstrate the existence of an MAE in remote test areas (remote MAE), then describe some of its temporal and spatial characteristics and compare them to those of the local MAE where adaptation and test areas are congruent. The results indicate that the remote MAE does indeed occur, but that it is weaker, more variable, and generally less tuned.

\section{GENERAL METHODS}

\section{Stimuli and apparatus}

For all experiments, a Macintosh II with a NuVista ${ }^{+}$image processing board was used to present the stimuli and record the responses. The adaptation stimulus consisted either of a sinusoidal grating or a plaid pattern (two superimposed sinusoidal gratings) which drifted within a circular window ( $5 \mathrm{deg}$ in diameter) during the adaptation period. The test stimulus was the phase-reversing grating described below which also appeared in a $5 \mathrm{deg}$ window that could either be placed at the same location as the adaptation window (local MAE) or at a location $6 \mathrm{deg}$ (center-to-center) below the adaptation window (remote MAE). All gratings had a contrast of 0.34 , with the average luminance of the gratings and the surrounding screen being $20 \mathrm{~cd} / \mathrm{cm}^{2}$. In the first three experiments, a low spatial frequency $(0.5 \mathrm{cpd})$ stationary sinusoidal horizontal grating (contrast of 0.8 ) filled the rest of the screen during the test period to enhance the aftereffect (Day and Strelow, 1971). In the center of the display, a fixation cross was presented continuously during adaptation and test (see Fig. 1, C and D).

\section{Phase-reversal test stimulus}

In all experiments, the test stimulus consisted of two superimposed sinusoidal gratings with the same orientation, but opposite directions of motion. Both had the same spatial frequency and drifted with the same velocity. When both gratings have the same contrast, this stimulus is ambiguous as to the direction of perceived motion (see Fig. 1A). But when the contrast of, for example, the downward grating is decreased, the stimulus seems to move upwards (see Fig. IB). In the experiments, the contrast of one of the two gratings could be adjusted by the observer from the original contrast of 0.34 in an ongoing way, up or down, with a step size of 0.02 . The observer's task was to try to keep the test stimulus from appearing to move in one particular direction by adjusting the contrast up or down. In this way, the effects of prior motion 
A

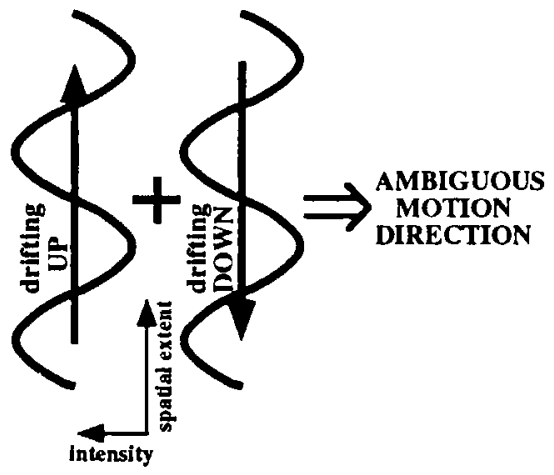

B

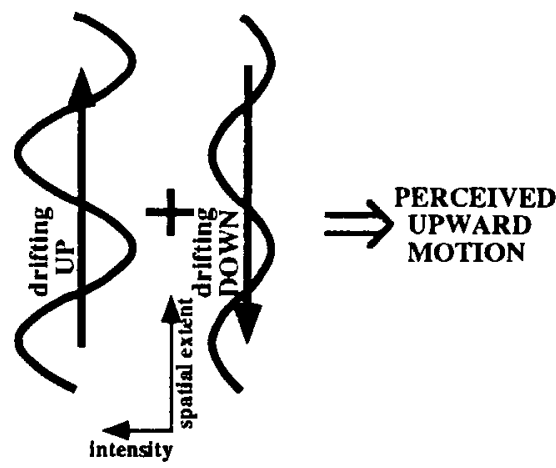

C

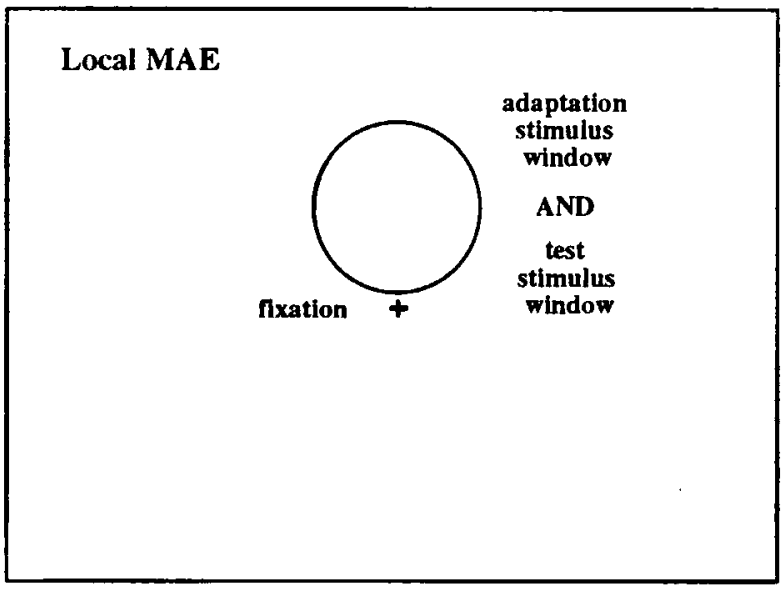

D

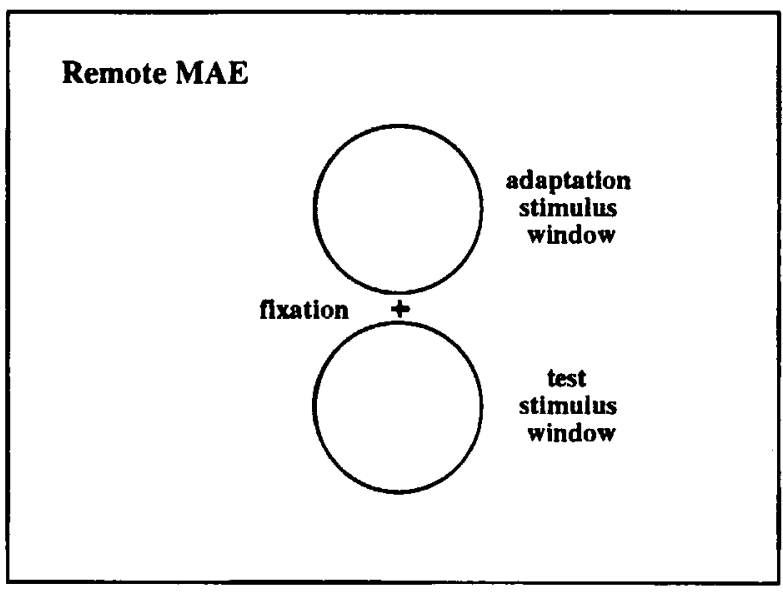

Figure 1. Schematic representations of the phase-reversal test stimulus and the display. A: Both components have the same contrast; motion direction is ambiguous. B: The downward drifting component has reduced contrast; perceived motion is upward. C: Display for the local MAE. Adaptation and test stimuli appear in the same location. D: Display for the remote MAE. Adaptation and test stimuli appear in different locations. 
adaptation could be cancelled continuously. The strength of the MAE was determined in the following way: The contrast settings of the adjustable grating of the test stimulus (sampling rate: $1 \mathrm{~Hz}$ ) were plotted as a function of time for the test period. The area between the adjusted contrast curve and the initial contrast level was taken as a measure of the strength of the obtained MAE (it was found that this measure correlated well with the maximum contrast adjustment). In the following, the results are, therefore, given in terms of 'MAE strength' in arbitrary units, based on the area under the adjustment curve.

\section{Procedure}

The observers were seated in front of the monitor at a distance of $57 \mathrm{~cm}$ with their head on a chin rest and maintained stable fixation of the cross during all the adaptation and test periods. At this distance, the screen had an extent of $23 \times 17 \mathrm{deg}$ of visual angle. They were free to initiate each trial when they were ready. Following adaptation, their task was to continuously cancel the MAE by adjusting the contrast of one of the component gratings of the phase-reversal test stimulus for as long as it was present.

The observers were one of the investigators and naive subjects recruited from undergraduate psychology classes. All had normal or corrected-to-normal vision.

\section{EXPERIMENT 1}

This experiment served several purposes. It is used here to demonstrate that an MAE can be found even when adaptation and test areas do not overlap. It is also used to show the time course of this remote MAE, and to compare it with that of the local MAE.

\section{Methods}

The adaptation stimulus was either a plaid pattern with 90 deg between the orientations of the two component gratings $( \pm 45 \mathrm{deg}$ from the horizontal) which had a spatial frequency of $1 \mathrm{cpd}$ each, or a horizontal grating ( $1 \mathrm{cpd})$. The drift rate for all gratings was set at $2 \mathrm{~Hz}$. The plaid and the grating were both perceived to move in a vertical direction (either up or down). Adaptation lasted for $30 \mathrm{~s}$ and was followed immediately by the presentation of the test stimulus.

The test stimulus was the phase-reversing grating described above with $1 \mathrm{cpd}$ gratings moving at $2 \mathrm{~Hz}$. It was always oriented horizontally (moving in a vertical direction) and could appear at either of the two positions described above (for the local and remote MAE, respectively). The test stimulus was presented for $60 \mathrm{~s}$. Though a longer time would have been preferable, the resulting fixation difficulties did not allow a longer presentation. Adaptation to upward and downward moving stimuli occurred in different sessions, but grating and plaid adapting stimuli as well as the local and remote arrangements were presented in random order in the same session.

\section{Results and discussion}

The time course of the local MAE obtained by adaptation to the plaid stimulus is graphed on the left of Fig. 2 for three observers, and corresponding results for the remote MAE are graphed for the same observers on the right. Each point is the average of six trials (plotted with \pm 1 standard deviation), three each for upward and 


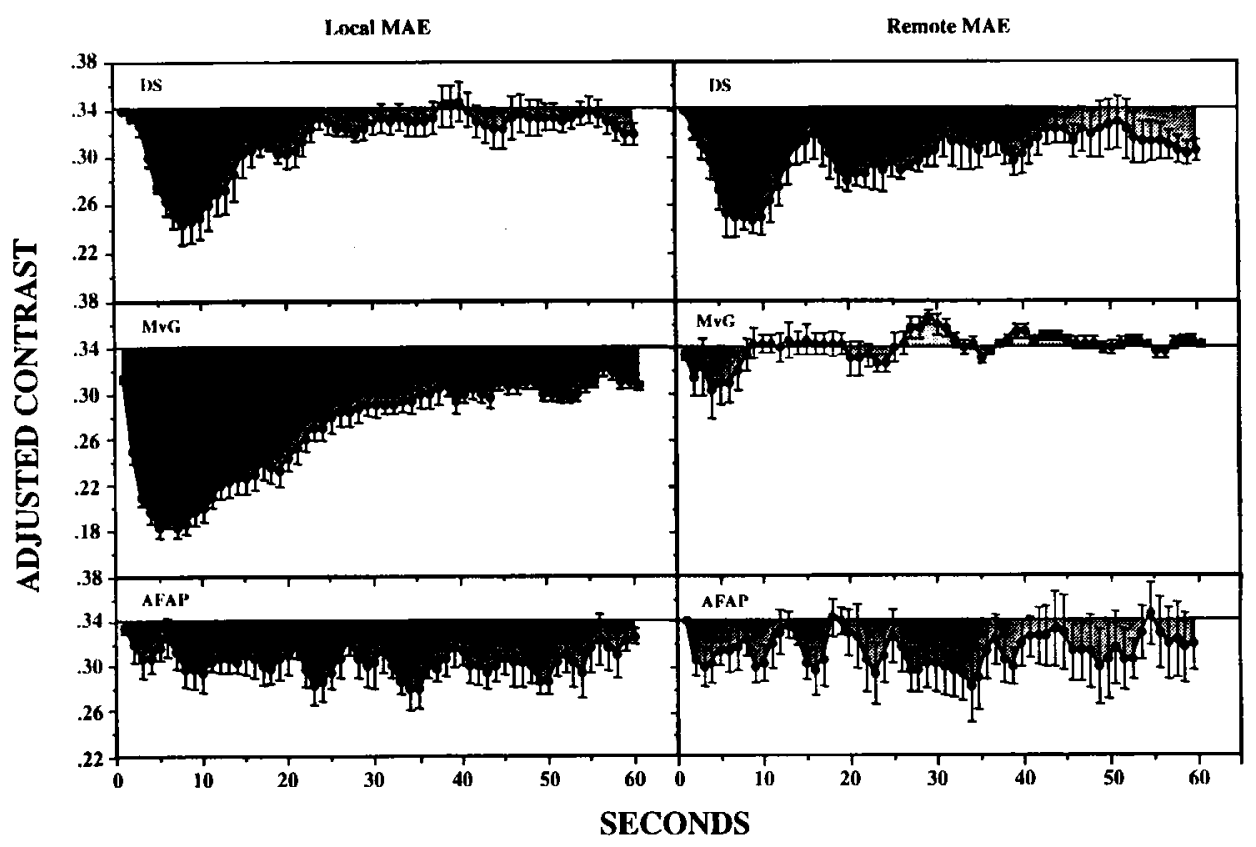

Figure 2. Examples from three observers of the time courses for local and remote MAEs.

downward adaptation. Combining the two directions also has the effect of cancelling potentially existing directional biases unrelated to any manipulations of the present experiment, like a priori preferences for up or down. In this representation, a downward deflection of the curve away from the original contrast line (contrast $=0.34$ ) indicates the cancellation of a negative aftereffect.

The local arrangement (left side), therefore, resulted in a strong, long-lasting MAE for all observers, although there were large individual differences. In some cases, the aftereffect had not dissipated after one minute of testing, though it had clearly diminished. Much smaller and more variable effects were found for the remote adaptation. Some observers showed a remote MAE similar in size and duration to the local MAE, while others had only small and brief, or even negligible effects. Similar results were also obtained for the grating adaptation stimulus (not shown here).

These results demonstrate that the time course and the size of MAEs can be measured in detail with the phase-reversal test stimulus, and that sizable MAEs exist both for the local and the remote presentation conditions. The individual differences may relate to how quickly and how well the cancellation can be achieved, which again may depend on the step size of the contrast adjustment.

\section{EXPERIMENT 2}

The phase-reversing grating measuring technique was used in this experiment to assess the directional tuning of both types of MAE (local and remote), again using grating and plaid adapting stimuli. Orientation tuning of the local MAE for grating stimuli has been previously reported by Over et al. (1973). They found fairly broad 
tuning of about $\pm 60 \mathrm{deg}$. With grating stimuli, orientation and direction are confounded because of the aperture problem, but since we also used plaid patterns we prefer to talk about directional tuning for all stimuli.

\section{Methods}

The adaptation stimuli were either a vertically drifting plaid pattern ( $60 \mathrm{deg}$ between the two sinusoidal component gratings, each oriented $60 \mathrm{deg}$ with respect to the horizontal) or a horizontal grating, also drifting vertically. All gratings had a spatial frequency of $1 \mathrm{cpd}$ and a drift rate of $2 \mathrm{~Hz}$. They were presented for $30 \mathrm{~s}$ for each trial. The phase reversing grating stimulus served as the test stimulus and was presented for $15 \mathrm{~s}$ immediately after each adaptation. This test stimulus could have directions that differed from the vertical by $0, \pm 20$, \pm 40 , or $\pm 60 \mathrm{deg}$. For $\pm 60 \mathrm{deg}$ it would thus be codirectional with one of the components of the plaid. Grating contrasts and adjustment step size were as in Experiment 1. Upward and downward moving adapting stimuli were presented in separate sessions. In each session, MAEs were measured for the local and remote arrangements, for both kinds of adaptation stimuli, and for all seven test stimulus directions. These 28 conditions were given four times each in random order.

\section{Results and discussion}

From the time course of the MAEs the overall strength of each MAE was estimated by calculating the area under the curve. This is plotted in Fig. 3 as a function of the difference between the drift directions of the adaptation and test stimuli. All points are the average of four observers for the plaid (A) and grating (B) adaptation stimuli. The directional tuning of the local MAE was very clear $\left(F_{6.18}=11.3 ; \mathbf{P}<0.00001\right.$ for the plaid; $F_{6,18}=4.4 ; \mathrm{P}<0.0065$ for the grating). The MAE was strongest when adaptation and test directions were the same. The MAE strength declined with direction difference and was still measurable at $\pm 60 \mathrm{deg}$ of direction difference. The situation was very different for the remote MAE. Here, the MAE was measurable, but its strength was much smaller over the whole range of direction difference. There was no obvious direction tuning $\left(F_{6,18}=1.2 ; \mathrm{P}>0.3\right.$ for plaid and grating) within the range of tested direction differences $(-60 \mathrm{deg}$ to $+60 \mathrm{deg})$, though one would expect to find a decline to near zero at direction differences of $\pm 90 \mathrm{deg}$. Adaptation with grating and plaid pattern gave results that were very similar in all the discussed points.

For the test directions of $\pm 60 \mathrm{deg}$, the orientations of the test grating and of one of the component gratings of the plaid pattern were the same. If the visual system treated the plaid in this instance as a combination of two moving gratings, one would expect strong aftereffects at these directions. This, however, did not occur, indicating that the plaid functioned as a coherent pattern in this case and not as a combination of two component gratings. The MAE thus was in response to the plaid and therefore cannot be localized at the level of area V1.

\section{EXPERIMENT 3}

In the previous experiment, the test stimulus direction was varied from trial to trial, while the adaptation direction was kept constant. This might have weakened the salience of the two component gratings, and thus no increased adaptation effect was found at the \pm 60 deg test directions. We investigated directional tuning again in 


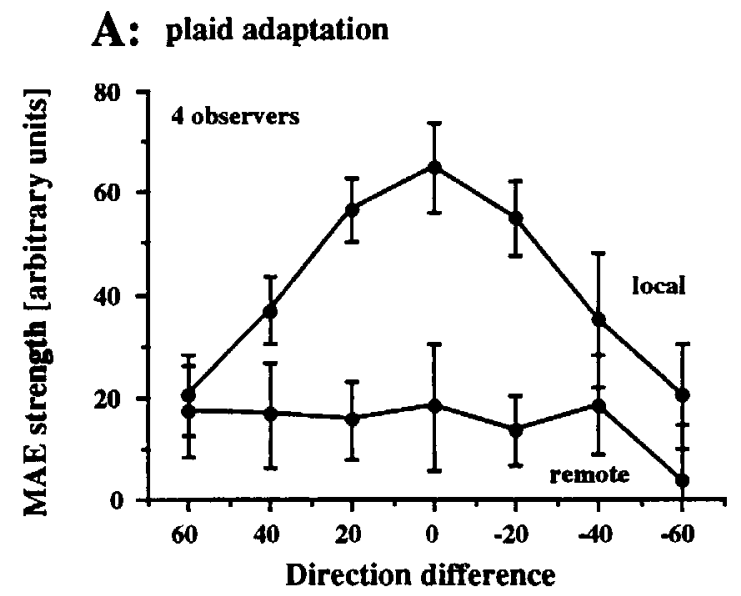

B: grating adaptation

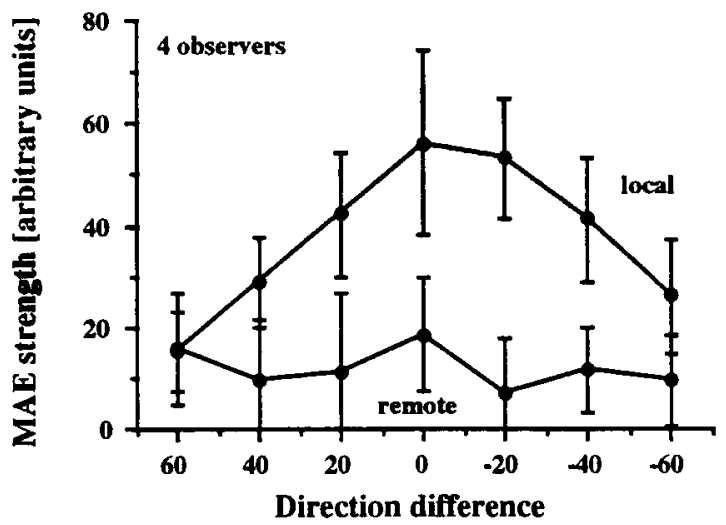

Figure 3. Strength of MAE (based on the area under the time course functions) for adaptation to a plaid (A) and a grating (B) as a function of the difference of direction between fixed adaptation and variable test stimulus. Local and remote effects are compared. Data are based on four observers in Expt 2.

another experiment where this time the test stimulus direction was kept constant, while the adaptation direction was varied.

\section{Methods}

As adaptation patterns, the same kind of plaid pattern was used as in the previous experiment $(60 \mathrm{deg}$ between the two sinusoidal component gratings, each with a spatial frequency of $1 \mathrm{cpd}$, drifting at $2 \mathrm{~Hz}$ ). The plaid was rotated, however, so that its drift direction subtended angles of $0, \pm 30$, or $\pm 60 \mathrm{deg}$ with respect to the vertical direction. Each adaptation episode lasted for $30 \mathrm{~s}$, after which the test stimulus was presented for $20 \mathrm{~s}$. There was only one test stimulus, the same horizontally oriented phase-reversing grating as before, which drifted in a vertical direction. Upward and downward moving adapting stimuli were presented in the same session. MAEs were 


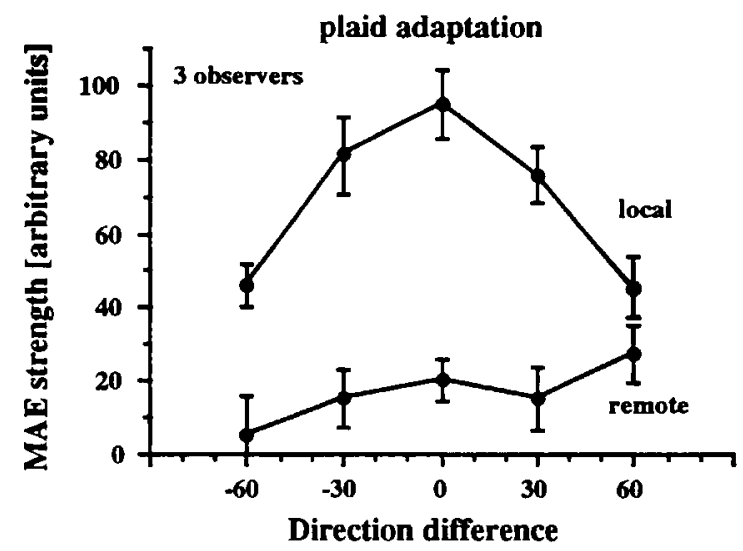

Figure 4. Directional tuning of local and remote MAE with adaptation to a plaid. Variable adaptation and fixed test stimulus. Data are based on three observers in Expt 3.

measured for the local and remote arrangements, and for all five adaptation stimulus directions. These 20 conditions were presented three times in random order.

\section{Results and discussion}

In Fig. 4, combined results obtained from three observers are presented for local and remote aftereffects for the plaid adaptation stimulus. As in the previous experiment, the local MAE showed clear directional tuning $\left(F_{4,8}=5.8 ; \mathrm{P}<0.025\right)$ with MAEs still strong at $\pm 60 \mathrm{deg}$ of direction difference. The remote MAE, on the other hand, did not change appreciably over the given range of direction difference $\left(F_{4,8}=1.5\right.$; $P>0.25$ ), and thus there was again no indication for directional tuning of the remote MAE beyond that necessary to give a directional bias. Similarly, there was no evidence that adaptation increased for a direction difference of $\pm 60 \mathrm{deg}$, i.e. for the case where the test grating and one of the component gratings of the adapting plaid pattern were collinear. This confirms our finding that the plaid was treated as a coherent pattern in these experiments and not as a combination of two component gratings. This is important for an understanding of the physiological mechanisms that may underlie the different MAEs.

\section{EXPERIMENT 4}

Another clue as to the nature of the underlying mechanisms may be found in the spatial frequency response of the two types of MAE. In the next experiment, therefore, we investigated the influence of spatial frequency on the strength of the MAE, using the same setup as in the previous experiments. We thus determined the spatial frequency tuning of local and remote MAEs.

\section{Methods}

Adapting stimuli were a vertically drifting plaid pattern with 90 deg between the two sinusoidal component gratings (1 cpd spatial frequency, $2 \mathrm{~Hz}$ drift rate), or a horizontal grating $(1 \mathrm{cpd}, 2 \mathrm{~Hz}$ ). They were presented for $30 \mathrm{~s}$. The test stimulus was a phase-reversing grating moving in a vertical direction and having various spatial frequencies $(0.25,0.5,0.75,1.0,1.5,2.0,4.0 \mathrm{cpd})$. Cancellation of the MAE was 
plaid adaptation

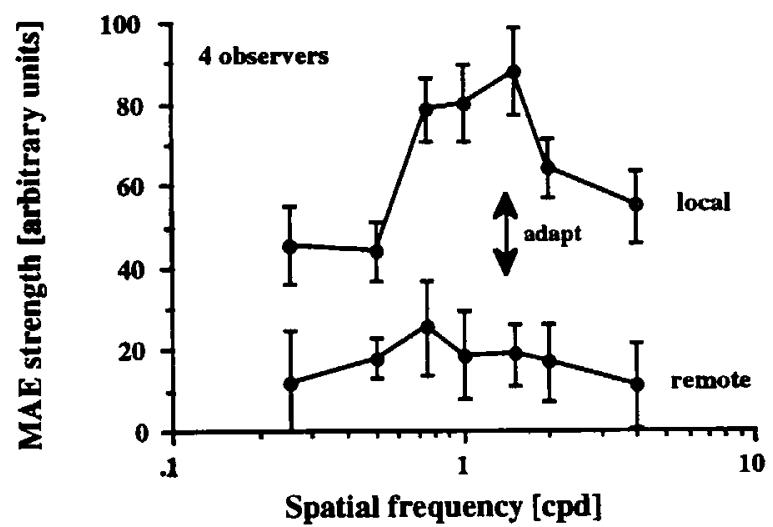

Figure 5. Spatial frequency tuning of local and remote MAE with adaptation to a plaid. Data are based on four observers in Expt 4.

measured for $20 \mathrm{~s}$. Again, both the local and the remote MAE conditions were presented in random order in the same session. Data were collected from four trials for each of the seven test stimuli. Upward and downward drifting adaptation stimuli were presented in separate sessions.

\section{Results and discussion}

The results for the strength of the MAE as a function of spatial frequency are graphed on a logarithmic scale in Fig. 5 for a group of four observers. Data for upward and downward drift directions were combined, so that each point is based on eight trials per observer. The local arrangement yielded a clear tuning function $\left(F_{6,18}=4.6\right.$; $P<0.005$ for the plaid adapting stimulus) that was centered at about the adapting frequency (which was the spatial period of the $90 \mathrm{deg}$ plaid, i.e. about $1.4 \mathrm{cpd}$ ). The remote arrangement, on the other hand, gave only a shallow tuning curve, slightly favoring lower spatial frequencies, but not significantly so $\left(F_{6,18}=1.4 ; \mathrm{P}>0.25\right)$. The results for the grating adaptation stimulus (not shown here) were similar in the sense that there was clear tuning for the local MAE, but basically no tuning effect for the remote condition.

\section{GENERAL DISCUSSION}

From this series of experiments, we want to draw four conclusions: (1) We designed and tested a new method of using a phase-reversal stimulus to cancel the MAE. It was shown to be useful and very sensitive, but somewhat unstable. (2) It was demonstrated that it is possible to show the existence of a negative motion aftereffect in a region that did not receive direct stimulation during adaptation. (3) The local MAE was shown to be most likely generated in a location at or beyond the pattern motion process. (4) The remote MAE showed no tuning for direction beyond the direction bias necessary for an MAE, and only little tuning for spatial frequency within the range of direction or spatial frequency differences that sufficed for the local MAE. 


\section{Methodological considerations}

The relative instability of the phase-reversal measuring technique is indicated by the large individual differences that were found for both kinds of MAE, but more so for the remote MAE. For many observers, the ambiguous test stimulus was perceived to move strongly in a direction opposite to the adaptation direction, as indicated by their large contrast adjustments to cancel this illusory motion. For some observers, however, the remote MAE was very small.

One factor contributing to the size of the individual differences may have been the step size of the contrast adjustment (0.02). For some observers, this was too small in the sense that they did not manage to cancel the remote MAE fast enough before it started to decay appreciably. Larger step sizes, on the other hand, made it difficult to precisely cancel the local MAE. Since the two effects were to be compared, a single step size was kept for all experimental conditions. With a more flexible choice of step size it might be possible to optimize both effects.

The present task required steady fixation for extended periods of time. The different abilities of observers to do this may have been another factor that contributed to the size of the individual differences. Eye movements by themselves, however, cannot be responsible for the remote MAE, since (as with the local MAE) in pilot experiments, corresponding effects were obtained with rotating rather than linearly drifting displays and with displays that consisted of two simultaneous motions in opposite directions.

In spite of these difficulties, the phase-reversal stimulus turned out to be extremely sensitive in revealing directional biases for very short adaptation times. This sensitivity may arise from the fact that this stimulus consists of two components that move in opposite directions and are both present simultaneously. In this way, neither of the directions is favored in the test stimulus. Not using a stationary test stimulus avoids the contradiction of seeing illusory motion, while at the same time noticing that location has not changed. For these reasons, the phase-reversal stimulus may have been more sensitive than the more conventional test stimuli.

The present results for the directional tuning can be compared with those obtained in previous studies (Over et al., 1973). The good correspondence between the previous and the present tuning functions speaks to the usefulness of the phase-reversal technique.

\section{Theoretical considerations}

The clear directional tuning of the local MAE elicited by adaptation to a plaid without appreciable effects at the directions of the components leads to the conclusion that the substrate for this MAE must be localized at or beyond the pattern motion process. This would preclude area V1 and suggest extrastriate areas such as MT or MST.

It is interesting to note, as was suggested by one of the reviewers of this article, that the results of the direction-tuning experiments also imply that the strong local MAE to a drifting grating will almost disappear when another grating with a different orientation or direction is added to form a drifting plaid pattern. The grating loses its identity and its MAE as it becomes part of the new pattern, the plaid. This is comparable to the analogous situation in physiological experiments by Movshon et al. (1985). There it was found that a V1 cell with the grating in its preferred orientation will not change its response if one adds another grating with a widely different 
orientation. On the other hand, some cells in MT (the pattern-motion cells) were reported to respond differently to the grating and to the plaid. It follows that the most likely substrate for the local MAE to plaid adaptation seems to be one consisting of pattern motion cells, which are found only in extrastriate cortical areas such as MT or MST.

When a grating was used as the adaptation stimulus, the obtained local MAE was very similar to that from a plaid moving in the same direction. This was true for the size of the effect and for its tuning curve. This similarity, as well as the existence of a similar remote MAE in both cases, suggests, furthermore, the possibility that the local MAE to gratings may also have an extrastriate locus.

The fact that for the plaid adaptation stimulus in the local arrangement no appreciable effects at directions corresponding to the plaid's component grating directions were obtained suggests further that the neural response to the coherent plaid pattern and the responses to the individual components are not simply serial stages of motion processing. Response to the pattern may inhibit the response to the individual components and vice versa (as suggested by Movshon et al., 1985). This corresponds to our perception: When the two gratings cohere and we see movement in the new direction, we no longer see the gratings move in the old directions.

The existence of a remote MAE also has implications for our understanding of the cortical mechanisms underlying the MAE in general. Here it is not possible to use the directional tuning of this effect, since it was not possible to demonstrate clear tuning functions for the remote MAE, at least not within the limits that were easily sufficient to show tuning for the local MAE. This wider tuning would be compatible with an extrastriate origin, but the remote effects were too small to allow us to specify the width of tuning beyond simple direction selectivity. But we can use the same kind of argumentation concerning RF size and layout that was used in the Introduction. This leads us to suggest that the remote MAE may also be localized at extrastriate sites: The present results can be understood in a scheme where the relevant RFs are much larger than those assumed for V1, and where the optimal stimuli may be much smaller than the size of the RF and placed anywhere within this large RF area. Thus a test stimulus can still strongly excite the same neuron, even if it is presented spatially separate from the adaptation area, but within the large RF. Neurons with such RF properties can be found in extrastriate areas in the primate visual system, specifically areas MT and MST, which have been implicated in the processing of motion (e.g. Maunsell and Van Essen, 1983; Albright, 1984; Mikami et al., 1986a, b; Tanaka et al., 1986; Maunsell and Newsome, 1987).

Our experiments are thus compatible with the view that adaptation to stimulus motion occurs at extrastriate locations for all the MAEs observed in the present study. The local and remote MAEs, however, are not equivalent in terms of their perceptual saliency or quality. This and the different tuning functions would suggest that different extrastriate mechanisms underlie the local and remote MAEs. This remains to be clarified in further experiments. The present results, however, suggest that patternselective cells in MT should be probed for adaptability to prolonged motion stimulation.

\section{Acknowledgments}

We thank Drs P. Cavanagh and P. Wenderoth for their critical reading of an earlier 
version of the manuscript. This research was supported by an NSERC operating grant (A-53) to MvG. Partial reports were presented at the Annual Meetings of ARVO, 1990 and SQRP, 1990.

\section{REFERENCES}

Albright, T. D. (1984). Direction and orientation selectivity of neurons in visual area MT of the macaque. J. Neurophysiol. 52, 1106-1130.

Anstis, S. M. and Duncan, K. (1983). Separate motion aftereffects from each eye and from both eyes. Vision Res. 23, 161-169.

Anstis, S. M. and Gregory, R. L. (1965). The after-effect of seen motion: the role of retinal stimulation and of eye movements. Q. J. Exp. Psych. 17, 173-174.

Day, R. H. and Strelow, E. (1971). Reduction or disappearance of visual aftereffect of movement in the absence of patterned surround. Nature 230, 55-56.

Lehmkuhle, S. W. and Fox, R. (1975). Effect of binocular rivalry suppression on the motion aftereffect. Vision Res. 15, 855-859.

Levinson, E. and Sekuler, R. (1975). The independence of channels in human vision selective for direction of movement. J. Physiol. (Lond.) 250, 347-366.

Maunsell, J. H. R. and Newsome, W. T. (1987). Visual processing in monkey extrastriate cortex. Ann. Rev. Neurosci. 10, 363-402.

Maunsell, J. H. R. and Van Essen, D. C. (1983). Functional properties of neurons in middle temporal visual area of the macaque monkey. I. Selectivity for stimulus direction, speed, and orientation. J. Neurophysiol. 49, 1127-1147.

Mikami, A., Newsome, W. T. and Wurtz, R. H. (1986a). Motion selectivity in macaque visual cortex: I. Mechanisms of direction and speed selectivity in extrastriate area MT. J. Neurophysiol. 55, 1308-1327.

Mikami, A., Newsome, W. T. and Wurtz, R. H. (1986b). Motion selectivity in macaque visual cortex: II. Spatio-temporal range of directional interactions in MT and V1. J. Neurophysiol. 55, 1328-1339.

Movshon, J. A., Adelson, E. H., Gizzi, M. S. and Newsome, W. T. (1985). The analysis of moving visual patterns. In: Pattern Recognition Mechanisms. C. Chagas, R. Gattass, and C. Gross (Eds). Vatican Press, Rome, pp. 117-151.

Over, R., Broerse, J., Crassini, B. and Lovegrove, W. (1973). Spatial determinants of the aftereffect of seen motion. Vision Res. 13, 1681-1690.

Tanaka, K., Hikosaka, K., Saito, H., Yukie, M., Fukada, Y. and Iwai, E. (1986). Analysis of local and wide-field movements in the superior temporal visual areas of the macaque monkey. J. Neurosci. 6 , 134-144.

von Grünau, M. W. (1986). A motion aftereffect for long-range stroboscopic apparent motion. Percept. Psychophys. 40, 31-38.

Weisstein, N., Maguire, W. and Berbaum, K. (1977). A phantom-motion aftereffect. Science 198, 955-958.

Wiesenfelder, H. and Blake, R. (1991). Apparent motion can survive binocular rivalry suppression. Vision Res. 31, 1589-1600.

Wohlgemuth, A. (1911). On the after-effect of seen movement. Br. J. Psychol. 1, 1-117. 